\title{
Apoptosis, Fibrosis and Senescence
}

\author{
Didier Portilla \\ Division of Nephrology, Department of Internal Medicine, University of Virginia, Charlottesville, Va., and \\ Salem Veterans Administration Medical Center, Salem, Va., USA
}

\section{Key Words}

Acute kidney injury - Apoptosis - Cellular senescence ·

Epithelial cell injury · Fibrosis - Proximal tubule metabolism important new therapeutic target. In this review, the current evidence and potential molecular mechanisms involved in the development of kidney fibrosis are discussed.

(c) 2014 S. Karger AG, Basel

\section{Abstract}

Fibrosis is a major hallmark of progressive kidney disease. The cellular mechanisms that lead to kidney tissue fibrosis are complex and include, for example, increased inflammation, increased oxidative stress, and proximal tubule cell death in the form of apoptosis or senescence. Recent studies have identified TWEAK, a tumor necrosis factor-like weak inducer of apoptosis, as a novel cytokine that mediates kidney inflammation in models of renal fibrosis. Inhibition of apoptosis via TWEAK inhibition has been shown to reduce kidney fibrosis. Recent studies using lineage tracing suggest that interstitial pericytes/perivascular fibroblasts differentiate into myofibroblasts and undergo proliferative expansion during fibrosis. Furthermore, increased expression of nuclear peroxisome proliferator-activated receptor-a in proximal tubules can directly reduce increased expression of transforming growth factor- $\beta 1$ and interstitial inflammation in models of renal fibrosis, which suggests preservation of proximal tubule cell metabolism and integrity represents an

\section{Introduction}

Acute kidney injury (AKI) affects as many as $5 \%$ of all patients who are hospitalized and it is now recognized as one of the strongest risk factors for the development of chronic kidney disease (CKD). Unequivocal data have indicated that AKI frequently does not resolve, and even when it does, it can often leave a reduced number of functioning nephrons that subsequently manifest as progressive CKD. It is clear that strategies to prevent AKI, treat $\mathrm{AKI}$, or inhibit the development of CKD as a result of $\mathrm{AKI}$ will have a major impact on the incidence of CKD in the community [1]. Currently, only therapies that target the

Targeting Recovery from Acute Kidney Injury: Round Table Conference at the 19th International Conference on Continuous Renal Replacement Therapies (Manchester Grand Hyatt, San Diego, Calif., USA, March 2-3, 2014).

\section{KARGER}

E-Mail karger@karger.com www.karger.com/nec
(C) 2014 S. Karger AG, Basel

$1660-2110 / 14 / 1274-0065 \$ 39.50 / 0$
Didier Portilla

Division of Nephrology, Department of Internal Medicine, University of Virginia West Complex MSB, 5th Floor, Room 5097 Charlottesville, VA 22908-0133 (USA)

E-Mail DP2BS@hscmail.mcc.virginia.edu 
Fig. 1. Mechanisms by which proximal tubule PPAR- $\alpha$ reduces renal fibrosis. TBM = Tubular basement membrane; UUO = unilateral ureteral obstruction.

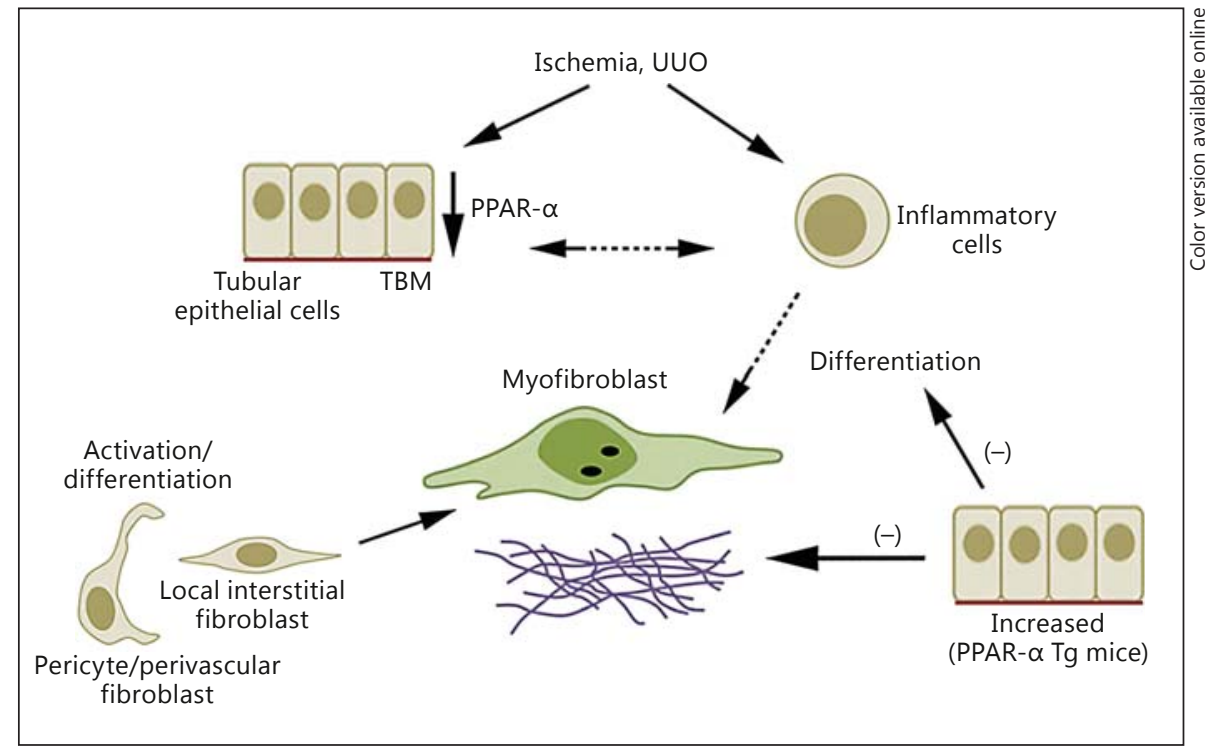

angiotensin receptor (angiotensin receptor 1 blockers or angiotensin-converting enzyme inhibitors) are used in clinical practice to slow the progression of CKD. There is, therefore, a pressing need for new therapies designed to treat or protect the damaged kidney.

\section{Epithelial Cell Injury as a Stimulus for Fibrosis}

Numerous studies have identified cell stress or cell injury in the tubule epithelial compartment, particularly the proximal tubule, as a stimulus for fibrosis [2, 3]. It is increasingly recognized that damaged or stressed epithelial cells exhibit a number of stereotyped responses that include endoplasmic reticulum stress, the unfolded protein response, apoptosis and necrosis, activation of epithelialto-mesenchymal transition genes, the activation of transforming growth factor- $\beta$, and cell cycle arrest, for example [3]. Proximal tubule cells are particularly dependent on the aerobic generation of high levels of ATP for cell survival and tissue health. Therefore, factors that affect or compromise ATP generation have a profound impact on epithelial cell function. Not only do injured and stressed epithelial cells fail to perform normal functions that are vital to renal function, they also generate a wide array of profibrotic and inflammatory factors that can drive the manifestations of CKD via cell-to-cell signaling mechanisms.

Although renal epithelial cell necrosis has long been regarded as the pathognomonic lesion of ischemic AKI, the amount of necrosis detected in human renal tissue fails to predict renal function, the potential for recovery of native renal function, or the need for renal replacement therapy in either native kidneys or allografts. The lack of correlation between classic cell necrosis and organ function implicates alternative forms of cell death as contributors to organ failure, and investigators have increasingly recognized that renal cell death also includes apoptosis, and autophagic and necroptotic cell death [4]. The morphological and biochemical characteristics of cells dying by apoptosis differ markedly from those of cells dying by necrosis. During apoptosis, cells decrease in size and round up. The nuclear chromatin undergoes condensation and fragmentation. The apoptotic cell then breaks apart into many plasma membrane-bound vesicles called 'apoptotic bodies', which contain fragments of condensed chromatin and morphologically intact organelles such as mitochondria. Apoptotic cells and bodies are rapidly phagocytosed, thereby protecting surrounding tissues from injury. The rapid and efficient clearance of apoptotic cells makes apoptosis extremely difficult to detect in tissue sections. Recent evidence in murine models of AKI suggests that apoptotic cell death correlates far better with the level of renal dysfunction than necrosis [5].

Apoptosis can be initiated by the binding of lethal ligands, such as FAS/CD95 ligand, tumor necrosis factor (TNF)- $\alpha$ and TNF (ligand) superfamily member 10 (best known as TNF-related apoptosis-inducing ligand), to various death receptors (i.e., FAS/CD95, TNF- $\alpha$ receptor 1 and TNF-related apoptosis-inducing ligand receptors 1 and 2, respectively). Renal tubular cells express these cell 
surface death receptors that upon activation during renal ischemia activate caspases and initiate apoptosis, which contribute to AKI [6]. This extrinsic apoptosis is a caspase-dependent cell death and hence can be suppressed by pan-caspase chemical inhibitors such as $\mathrm{N}$-benzyloxycarbonyl-Val-Ala-Asp-fluoromethylketone or by the overexpression of viral inhibitors of caspases like cytokine response modifier A. Pro- and anti-apoptotic signals converge at mitochondrial membranes, which become permeabilized when the former predominate over the latter. Mitochondrial outer membrane permeability can start at the outer mitochondrial membrane owing to the pore-forming activity of proapoptotic members of the Bcl-2 protein family such as Bak and Bax or can result from a phenomenon (the so-called mitochondrial permeability transition) that originates at the inner mitochondrial membrane due to the opening of a multiprotein complex known as the permeability transition pore complex. In a recent study, Wei et al. [7] examined the role of Bax and Bak in tubular cell apoptosis during ischemic AKI using several gene knockout models. They found that global knockout of Bak protected mice from ischemic AKI with improved renal function. Bax and Bak knockout blocked kidney tubular apoptosis without affecting tubular necrosis. In addition, Bak deficiency prevented mitochondrial fragmentation in ischemic AKI. These studies support a critical role of Bax and Bak in tubular cell apoptosis in ischemic AKI.

Intrinsic tubular cell apoptosis due to caspase-dependent or independent pathways contributes to ischemic as well as nephrotoxic AKI. In this condition, Bax and Bak are activated and form oligomers in mitochondria, which leads to permeabilization of the outer membrane and release of apoptogenic factors such as cytochrome c. Brooks et al. [8] examined the mechanism of mitochondrial damage during AKI. Following ATP depletion or cisplatin treatment of tubular cells, they observed mitochondrial fragmentation prior to cytochrome c release and apoptosis. This mitochondrial fragmentation was inhibited by Bcl-2 but not by caspase inhibitors. Dynamin-related protein (Drp1), a critical mitochondrial fission protein, translocated to mitochondria early during tubular cell injury, and both siRNA knockdown of Drp1 and expression of a dominant negative Drp1 attenuated mitochondrial fragmentation, cytochrome $\mathrm{c}$ release, caspase activation, and apoptosis. This study demonstrates a rapid regulation of mitochondrial dynamics during AKI and identifies mitochondrial fragmentation as a novel mechanism contributing to mitochondrial damage and apoptosis in vivo in mouse models of AKI.

Apoptosis, Fibrosis and Senescence

\section{Role of TWEAK-Fn14 in AKI and CKD}

TWEAK, a TNF-like weak inducer of apoptosis, a type II transmembrane protein and member 12 of the TNF superfamily, was discovered in 1997. Its receptor, Fn14, a type I transmembrane protein and member of the fibroblast growth factor-inducible gene family, was recognized by differential display and later identified in 2001 as the TWEAK receptor. In kidney tissue, Ortiz and his group $[9,10]$ have presented ample evidence to support the role of TWEAK activation of intrinsic renal cell Fn14 receptors in the pathogenesis of AKI and CKD. Increased expression of TWEAK and Fn14 has been observed in human and experimental models of tubulointerstitial kidney disease. Upregulation of tubular cell Fn14 was seen within $24 \mathrm{~h}$ in experimental models of AKI induced by folic acid or by renal ischemia-reperfusion (IR) injury. Kidney TWEAK was also increased in these same models but to a lesser extent than Fn14. Increased tubular Fn14 has also been observed in human ischemic AKI and in acute or chronic human tubulointerstitial inflammation associated with glomerular injury or caused by acute tubulointerstitial nephritis. Targeting TWEAK with neutralizing anti-TWEAK antibodies, the use of TWEAK knockout mice or targeting Fn 14 by blocking anti-Fn 14 antibodies improved histological and functional features of AKI. Based on the existent evidence of beneficial effects of TWEAK targeting, a clinical trial is exploring the contribution of TWEAK to lupus nephritis. In addition, a potential role of TWEAK in kidney regeneration should be explored.

\section{Senescence and CKD}

Cellular senescence is defined as a stable cell cycle arrest elicited in response to multiple types of damage, such as intense oncogenic signaling, DNA damage, and telomere loss [11]. Senescence cells are found in association with aging, tissue repair, and cancer. More recently, programmed cell senescence was documented during mammalian embryonic development. The most widely used senescence marker is senescence-associated $\beta$-galactosidase activity, which likely reflects the increased autophagy occurring in senescent cells. Other canonical senescence markers include p53, p21, p16, and reduced RB phosporylation, which collectively mediate the phenotypic changes associated with cell cycle arrest. A recent study documented the presence of senescence cell death during the development of CKD [12]. These investigators 
used young (8-10 weeks) and aged (46-49 weeks) mice and subjected them to 30 -min bilateral IR to induce AKI. The aged animals had greater mortality and prolonged elevation in serum creatinine correlating with less epithelial cell proliferation compared to the young. Six weeks after reperfusion, interstitial fibrosis was greater in aged kidneys based on picrosirius red staining and increased extracellular matrix genes. Six weeks after reperfusion, aged kidneys also expressed higher levels of p53 and p21 compared to the young, correlating with greater increases in senescence-associated $\beta$-galactosidase, a known marker of cellular senescence. In addition, increased influx of macrophages and CD4 T cells was also seen in aged mice, which was accompanied by increases in mRNA levels of TNF- $\alpha$ and monocyte chemoattractant protein 1 . The authors also found reduced microvascular density, correlating with increased nitrotyrosine, a marker of oxidative stress. The findings of this study as well as previous studies [13] suggest that CKD is a chronic condition that promotes cellular senescence and premature aging. This occurs through several mechanisms, including DNA and mitochondria damage, increased reactive oxygen species generation, persistent inflammation, phosphate toxicity, decreased khloto expression, and telomere attrition. Because the recent literature suggests that growth factor signaling has an important role in the aging phenotype, interventions targeting the nutrient-sensing mTOR pathway, such as rapamycin and resveratrol, are of major interest in studies of uremic premature aging. Given the combined role of phosphate and klotho in uremic vascular disease, interventions that target these factors should also be considered.

\section{The Role of Fibrosis in Disease Progression}

Recent studies, primarily from animal models of kidney disease, have identified a separate lineage of cells derived from the mesenchyme in the normal kidney that are termed 'pericytes' or 'resident fibroblasts'. These cells represent $>5 \%$ of normal kidney cells and perform critical homeostatic and regenerative functions, particularly with respect to microvascular homeostasis [14]. In CKD, these pericytes and resident fibroblasts become activated as myofibroblasts. Myofibroblasts are contractile cells that deposit the pathological fibrillar matrix, often described as fibrosis or scar tissue, in the renal cortex and medulla. Myofibroblasts in the glomerulus deposit a pathological fibrous matrix known as mesangial matrix expansion or mesangial nodules, and these contribute to glomerulo- sclerosis when combined with the loss of glomerular capillaries. Fibrosis is a pathological fibrillar matrix that accumulates in the virtual space between capillaries and tubules of nephrons, or around glomerular capillaries. It obliterates local structures. In the glomerulus, it frequently accumulates initially in the mesangial area, along the capillary loop itself, or in the proliferation of cells that occupy the urinary space, often known as a 'crescent'. The fibrotic material encroaches on capillaries and prevents them from functioning. Fibrosis per se reduces nephron function and promotes tissue ischemia, leading to distortion of the normal tissue architecture. However, myofibroblasts, the activated pericytes, are contractile and can distort the tissue architecture. The myofibroblast is also an inflammatory cell that secretes innate immune cytokines, chemokines, and oxygen radicals. Furthermore, the myofibroblast can no longer perform the normal functions of the pericyte. Pericytes normally 'nurse' capillaries and support microvascular stability. The loss of these functions leaves unstable capillaries that are prone to ineffective angiogenesis, increased permeability, and often capillary demise, known as capillary rarefaction. Therefore, myofibroblasts cause fibrosis, are a source of inflammation, and promote the loss of capillaries. The myofibroblast is therefore a major new target for novel therapeutics in kidney diseases.

\section{Importance of Preserving Proximal Tubule Cell Metabolism during AKI}

The renal cortex and, more specifically, the proximal tubule depend heavily on fatty acids as a major source of energy. Fatty acids delivered to renal epithelial cells are primarily used for energy production by mitochondria and peroxisomes. We have described that the process of fatty acid oxidation in peroxisomes and mitochondrial compartments is inhibited in freshly isolated proximal tubules subjected to hypoxic injury and in vivo in the renal tissue of animals subjected to IR injury and cisplatininduced AKI. This results in the accumulation of longchain free fatty acids and long-chain acylcarnitines. In addition, fatty acid accumulation during AKI is also accompanied by the accumulation of triglycerides and cholesterol as part of a renal stress response. Recently, we generated proximal tubule peroxisome proliferator-activated receptor (PPAR)- $\alpha$ Tg mice, which showed a protective effect in the proximal tubule by the activation of mitochondrial and peroxisomal fatty acid oxidation in the IR injury and cisplatin models of AKI. In models of 
renal fibrosis, using the model of unilateral ureteral obstruction, we demonstrated a significant reduction in proximal tubule cell death and reduced apoptosis accompanied by a reduction in extracellular components in the tubulointerstitial space in PPAR- $\alpha$ Tg mice compared to wild-type mice [15]. These studies suggest that improving proximal tubule fatty acid oxidation represents a novel downstream therapeutic target to ameliorate progression of CKD.

\section{Acknowledgment}

The work was supported by NIH UAB-UCSD O'Brien Center grant P30 DK079337. Furthermore, D. Portilla's laboratory is funded by NIH grant DK75976 and a Veterans Affairs Merit award.

\section{References}

1 Leung KC, Tonelli M, James MT: Chronic kidney disease following acute kidney injury - risk and outcomes. Nat Rev Nephrol 2013;9:77-85.

2 Bonventre JV, Yang L: Cellular pathophysiology of ischemic acute kidney injury. J Clin Invest 2011;121:4210-4221.

3 Friedman SL, Sheppard D, Duffield JS, Violette S: Therapy for fibrotic diseases: nearing the start line. Sci Transl Med 2013;5:167sr1.

4 Linkermann A, Brasen JH, Darding M, Jin MK, Sanz AB, Heller JO, De Zen F, Weinlich R, Ortiz A, Walczak H, Weinberg JM, Green DR, Kunzendorf U, Krauwald S: Two independent pathways of regulated necrosis mediate ischemia-reperfusion injury. Proc Natl Acad Sci USA 2013;110:12024-12029.

5 Havasi A, Borkan SC: Apoptosis and acute kidney injury. Kidney Int 2011;80:29-40.

6 Galluzzi L, Vitale I, Abrams JM, et al: Molecular definitions of cell death subroutines: recommendations of the Nomenclature Committee on Cell Death 2012. Cell Death Differ 2012;19:107-120.
Wei Q, Dong G, Chen J-K, Ramesh G, Dong $\mathrm{Z}$ : Bax and Bak have critical roles in ischemic acute kidney injury in global and proximal tubule-specific knockout mouse models. Kidney Int 2013;84:138-148.

8 Brooks C, Wei Q, Cho S-G, Dong Z: Regulation of mitochondrial dynamics in acute kidney injury in cell culture and rodent models. J Clin Invest 2009;119:1275-1285.

-9 Sanz AB, Izquierdo MC, Sanchez Nino MD, Ucero AC, Egido J, Ruiz-Ortega M, Ramos AM, Putterman C, Ortiz A: TWEAK and the progression of renal disease: clinical translation. Nephrol Dial Transplant 2014;29:154162.

10 Ucero AC, Benito-Martin A, Izquierdo MC, Sanchez-Nino MD, Sanz AB, Ramos AM, Berzal S, Ruiz-Ortega M, Egido J, Ortiz A: Unilareal ureteral obstruction: beyond obstruction. Int Urol Nephrol 2014;46:765776.

11 Campisi J, d’Adda di Fagagna F: Cellular senescence: when bad things happen to good cells. Nat Rev Mol Cell Biol 2997;8:729-740.
12 Clements ME, Chaber CJ, Ledbetter SR, Zuk A: Increased cellular senescence and vascular rarefaction exacerbate the progression of kidney fibrosis in aged mice following transient ischemic injury. PLoS One 2013:8:e70464.

13 Stenvinkel P, Larson TE: Chronic kidney disease: a clinical model of premature aging. Am J Kidney Dis 2013:62:339-351.

14 Humphreys BD, Lin SL, Kobayashi A, Hudson TE, Nowlin BT, Bonventre JV, Valerius MT, McMahon AP, Duffield JS: Fate tracing reveals the pericyte and not epithelial origin of myofibroblasts in kidney fibrosis. Am J Pathol 2010;176:85-97.

15 Li S, Mariappan N, Megyesi J, Shank B, Kannan K, Theus S, Price PM, Duffield JS, Portilla D: Proximal tubule PPARa attenuates renal fibrosis and inflammation caused by unilateral ureteral obstruction. Am J Physiol Renal Physiol 2013;305:F618-F627. 